\title{
Compounds from Terminalia mantaly L. (Combretaceae) Stem Bark Exhibit Potent Inhibition against Some Pathogenic Yeasts and Enzymes of Metabolic Significance
}

\author{
Marthe Aimée Tchuente Tchuenmogne ${ }^{1}$, Thierry Ngouana Kammalac ${ }^{2}$, Sebastian Gohlke ${ }^{3}$, \\ Rufin Marie Toghueo Kouipou ${ }^{2}$, Abdulselam Aslan ${ }^{4}$, Muslum Kuzu ${ }^{5}$, Veysel Comakli ${ }^{6}$, \\ Ramazan Demirdag ${ }^{6}$, Silvère Augustin Ngouela ${ }^{1}$, Etienne Tsamo ${ }^{1}$, Norbert Sewald ${ }^{3}$, \\ Bruno Ndjakou Lenta ${ }^{7, *}$ and Fabrice Fekam Boyom ${ }^{2, *}$ \\ 1 Laboratory of Natural Products and Organic Synthesis, Department of Organic Chemistry, \\ Faculty of Science, University of Yaoundé 1, P.O. Box 812, Yaoundé, Cameroon; \\ tch_aimee@yahoo.fr (M.A.T.T.); sngouela@yahoo.fr (S.A.N.); tsamoet@yahoo.fr (E.T.) \\ 2 Antimicrobial \& Biocontrol Agents Unit, Laboratory for Phytobiochemistry and Medicinal Plants Studies, \\ Department of Biochemistry, Faculty of Science, University of Yaoundé I, P.O. Box 812, Yaoundé, Cameroon; \\ ngouanathi@yahoo.com (T.N.K.); toghueo.rufin@yahoo.fr (R.M.T.K.) \\ 3 Chemistry Department, Organic and Bioorganic Chemistry, Bielefeld University, P.O. Box 100131, \\ D-33501 Bielefeld, Germany; sebastian.gohlke@uni-bielefeld.de (S.G.); \\ norbert.sewald@uni-bielefeld.de (N.S.) \\ 4 Faculty of Engineering, Department of Industrial Engineering, Giresun University, 28200 Giresun, Turkey; \\ abdulselam@hotmail.de \\ 5 Faculty of Pharmacy, Department of Basic Pharmaceutical Sciences, Agrı Ibrahim Cecen University, \\ 04100 Agri, Turkey; mkuzu@agri.edu.tr \\ 6 School of Health, Department of Nutrition and Dietetics, Agrı Ibrahim Cecen University, 04100 Agri, Turkey; \\ veysel_comakli@hotmail.com (V.C.); r.demirdag@hotmail.com (R.D.) \\ 7 Department of Chemistry, Higher Teacher Training College, University of Yaoundé 1, Yaoundé, Cameroon \\ * Correspondence: lentabruno@yahoo.fr (B.N.L.); fabrice.boyom@fulbrightmail.org (F.F.B.); \\ Tel.: +237-675-097-561 (B.N.L.); +237-677-276-585 (F.F.B.)
}

Academic Editor: James D. Adams

Received: 1 November 2016; Accepted: 12 January 2017; Published: 24 January 2017

\begin{abstract}
Background: Pathogenic yeasts resistance to current drugs emphasizes the need for new, safe, and cost-effective drugs. Also, new inhibitors are needed to control the effects of enzymes that are implicated in metabolic dysfunctions such as cancer, obesity, and epilepsy. Methods: The anti-yeast extract from Terminalia mantaly (Combretaceae) was fractionated and the structures of the isolated compounds established by means of spectroscopic analysis and comparison with literature data. Activity was assessed against Candida albicans, C. parapsilosis and C. krusei using the microdilution method, and against four enzymes of metabolic significance: glucose-6-phosphate dehydrogenase, human erythrocyte carbonic anhydrase I and II, and glutathione $S$-transferase. Results: Seven compounds, 3,3'-di-O-methylellagic acid 4'-O- $\alpha$-rhamnopyranoside; 3-O-methylellagic acid; arjungenin or 2,3,19,23-tetrahydroxyolean-12-en28-oї acid; arjunglucoside or 2,3,19,23-tetrahydroxyolean-12-en-28-oïc acid glucopyranoside; $2 \alpha, 3 \alpha, 24$-trihydroxyolean-11,13(18)-dien-28-oïc acid; stigmasterol; and stigmasterol 3-O- $\beta$-dglucopyranoside were isolated from the extract. Among those, 3,3'-di-O-methylellagic acid $4^{\prime}$-O- $\alpha$-rhamnopyranoside, 3-O-methylellagic acid, and arjunglucoside showed anti-yeast activity comparable to that of reference fluconazole with minimal inhibitory concentrations (MIC) below $32 \mu \mathrm{g} / \mathrm{mL}$. Besides, Arjunglucoside potently inhibited the tested enzymes with 50\% inhibitory concentrations $\left(\mathrm{IC}_{50}\right)$ below $4 \mu \mathrm{M}$ and inhibitory constant $(\mathrm{Ki})<3 \mu \mathrm{M}$. Conclusions: The results achieved indicate that further SAR studies will likely identify potent hit derivatives that should subsequently enter the drug development pipeline.
\end{abstract}


Keywords: Terminalia mantaly; Combretaceae; anti-yeast; enzyme inhibitors

\section{Introduction}

Fungal diseases affect 3-4 million people worldwide every year. Of particular importance, the increasing resistance of pathogenic opportunistic yeasts to current drugs is a serious concern and has attracted the attention of the scientific community. New, safe, and cost-effective drugs of natural or synthetic origin are therefore actively being researched [1]. Recent epidemiological data highlight the increasing burden of pathogenic yeasts on people in poor settings [2-4]. Candida species and Cryptococcus neoformans are the major pathogenic yeasts and only a few antifungal drugs have been developed so far to treat the invasive infections they cause [5,6]. Medicinal plants have shown credibility as sources of treatment for infectious diseases [7]. In Cameroon, extracts from medicinal plants such as Terminalia mantaly (Combretaceae) are widely used by traditional healers to control diverse infections or associated symptoms, including but not limited to dysentery, gastroenteritis, hypertension, diabetes, and oral, dental, cutaneous and genital affections [8]. Previous studies on the extracts of this plant have showed antibacterial and antifungal properties, but their chemical compositions have not yet been determined [9]. However, phytochemical studies of other species of the genus Terminalia have reported the presence of flavonoids, terpenoids and their glycosides derivatives, tannins, flavonones and chalcones [10-18]. In spite of the work done on Terminalia species, no investigation has been attempted yet on the enzyme inhibition properties of their extracts and constituents targeting glucose-6-phosphate dehydrogenase, carbonic anhydrase and glutathione $S$-transferase.

Glucose-6-phosphate dehydrogenase (G6PD; EC 1.1.1.49) is an enzyme that catalyzes the reaction of glucose-6-phosphate into phosphogluconate, which is the rate-limiting first step of the pentose phosphate pathway. The end products of this pathway are ribose-5-phosphate and NADPH. Ribose-5-phosphate is used in DNA or RNA synthesis in cell reproduction, and NADPH is used as a coenzyme for the enzymes participating in the production of reduced glutathione. Given its role in cell growth, this enzyme is of high importance to mammal cells $[19,20]$. However, several studies have shown that this enzyme has an important role in the pathology of some diseases like cancer, hypertension, heart failure and type 2 diabetes. G6PD activity increases in cancer cells and its inhibition results in decreased cell proliferation and induction of apoptosis. For example, 6-aminonicotinamide, which is an inhibitor of G6PD, has found use in the therapy of various tumors in the past [21].

The carbonic anhydrase (CA; carbonate hydro-lyase, EC 4.2.1.1) enzyme exists commonly in living organisms, and has various isoenzymes according to conditions and necessities of the medium. It is one of the most studied enzymes and CAI and CAII are the most common isoenzymes [22]. In many physiological and pathological processes, CAs catalyze the conversion of $\mathrm{CO}_{2}$ to $\mathrm{HCO}_{3}{ }^{-}$and $\mathrm{H}^{+}$. In addition, CA inhibitors may be used in the treatment of various diseases such as oedema, glaucoma, obesity, cancer, epilepsy and osteoporosis [23].

In living cells, the deleterious effects of free radicals and their intermediates are eliminated or minimized by various enzymatic and non-enzymatic defense systems. Enzymatic defense is provided by several enzymes such as glutathione $S$-transferase (GST), glutathione reductase, glutathione peroxidase, superoxide dismutase, and catalase [24]. The GSTs (EC 2.5.1.18) are a group of multifunctional enzymes that play an important role in animal metabolism [25]. GSTs are important for the fight against cancer because of their interactions with carcinogens and chemotherapeutic agents. They are the target of antiasthmatic and antitumor drugs [26]. Production of excessive amounts of GST in mammalian tumor cells leads to resistance to some anticancer drugs and chemical carcinogens [27].

The reduction of drug effects in tumor cells is an important factor limiting the therapeutic efficacy of an antineoplastic agent. Over time, the development of this resistance is associated with glutathione (GSH) and glutathione S-transferase (GST) levels in cells and changes in permeability to the drug. In 
this regard, G6PD, CA I, II or GST inhibitors may be useful because of their several applications, in particular for the treatment of glaucoma, epilepsy, cancer and as diuretics.

In our search for bioactive secondary metabolites from Cameroonian medicinal plants, we have investigated the $\mathrm{MeOH}$ extract of the stem bark of T. mantaly L. (Combretaceae) that previously showed anti-yeast activity. We report in this paper the inhibitory potential of compounds isolated from this extract against some pathogenic yeasts and some enzymes of metabolic significance.

\section{Materials and Methods}

\subsection{General Experimental Procedures}

The physicochemical analyses of the isolated natural products were essentially performed as previously described [28]. Optical rotations were measured on a JASCO digital polarimeter (model DIP-3600, JASCO, Tokyo, Japan). UV spectra were determined on a Spectronic Unicam spectrophotometer (Thermo Scientific, Waltham, MA, USA). IR spectra were determined on a JASCO Fourier transform IR-420 spectrometer (JASCO, Tokyo, Japan). ${ }^{1} \mathrm{H}$ and ${ }^{13} \mathrm{C}$ NMR spectra were run on a Bruker spectrometer (Bruker Corporation, Brussels, Belgium) equipped with $5 \mathrm{~mm}{ }^{1} \mathrm{H}$ and ${ }^{13} \mathrm{C}$ probes operating at 500 and $125 \mathrm{MHz}$, respectively, with Tetramethylsilane (TMS) as internal standard. Silica gel 230-400 mesh (Merck, Bielefeld, Germany) and silica gel 70-230 mesh (Merck) were used for flash and column chromatography while precoated aluminum-backed silica gel $60 \mathrm{~F} 254$ sheets were used for TLC. Spots were visualized under UV light (254 and $365 \mathrm{~nm}$ ) or using $\mathrm{MeOH}-\mathrm{H}_{2} \mathrm{SO}_{4}$ reagent.

\subsection{Plant Material}

The stem bark of T. mantaly (Combretaceae) was collected in Yaoundé, Cameroon in May 2012 and identified at the Cameroon National Herbarium where a voucher specimen is deposited under the reference $\mathrm{N}^{\circ} 64212 / \mathrm{HNC}$ (T. mantaly $\mathrm{H}$. Perrier).

\subsection{Microbial Isolates}

Yeast isolates were kindly provided by the Laboratory of Clinical Biology, Yaoundé Central Hospital and consisted of clinical isolates of C. albicans, C. krusei and C. parapsilosis. Yeasts were maintained at room temperature and cultured at $37^{\circ} \mathrm{C}$ for $24 \mathrm{~h}$ on Sabouraud Dextrose Agar (Oxoid, Drongen, Belgium) slants prior to use.

\subsection{Plant Extraction and Screening of Anti-Yeast Activity}

The harvested T. mantaly stem bark was dried at room temperature and ground using a blender. The powdered stem bark $(7 \mathrm{~kg})$ was extracted at r.t. with $\mathrm{MeOH}(48 \mathrm{~h})$. The extract was concentrated under vacuum to afford a dark residue ( $250 \mathrm{~g})$. Minimal inhibitory concentration (MIC) of the extract was determined according to the CLSI M27-A3 [6] protocol with little modifications. The RPMI 1640 supplemented with $2 \%$ glucose was used as culture medium. Briefly for the fungal susceptibility tests, $50 \mu \mathrm{L}$ of serially two-fold diluted concentrations of the crude extract were added in triplicate wells of a 96-wells microtiter plate. Fifty $\mu \mathrm{L}$ of fungal inocula standardized to a final concentration of $0.5-2.5 \times 10^{3} \mathrm{CFU} / \mathrm{mL}$ were then individually added in each well of the plate. Plant crude extract and the positive control (fluconazole) at concentrations of 0.12 to $64 \mu \mathrm{g} / \mathrm{mL}$ were tested in a final volume of $100 \mu \mathrm{L}$. So-prepared plates were incubated at $37^{\circ} \mathrm{C}$ for $48 \mathrm{~h}$. MIC value was subsequently determined through macroscopic observation of plate wells, and was defined as the lowest concentration of the inhibitor that allowed no visible growth of the microorganism after overnight incubation compared to the growth control. 


\subsection{Isolation of Compounds and Screening for Activity}

A portion of $180 \mathrm{~g}$ of the extract was subjected to medium pressure flash chromatography over silica gel (Merck, 70-230 mesh) using mixtures $n$-hexane-EtOAc of increasing polarity ((70:30)-(0:100)) and EtOAc-MeOH ((95:5)-(50:50)), resulting in the collection of 75 fractions of $500 \mathrm{~mL}$ each, which were combined on the basis of TLC analysis to create four fractions labeled $\mathrm{T}_{1}-\mathrm{T}_{4}$. Fraction $\mathrm{T}_{1}(m=14.4 \mathrm{~g})$ obtained from the mixtures of $n$-hexane-EtOAc (100:0 to 70:30) was subjected to silica gel column chromatography, eluted with $n$-hexane-EtOAc, and yielded oils stigmasterol $(23 \mathrm{mg})$ and arjungenin $(7 \mathrm{mg})$. From fraction $\mathrm{T}_{2}(m=60.3 \mathrm{~g})$, eluted with $n$-hexane-EtOAc ((50:50)-(25:75)), stigmasterol

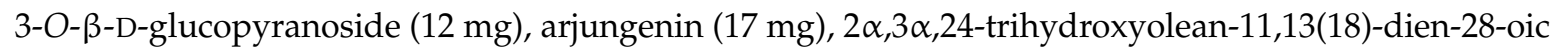
acid $(5.0 \mathrm{mg})$ and arjunglucoside $(6 \mathrm{mg})$ were isolated. Column chromatography of fraction $\mathrm{T}_{3}$ $(m=55.0 \mathrm{~g})$ on silica gel and eluted with the mixtures of EtOAc-MeOH ((100:0)-(85:15)), yielded 3,3'-di-O-methylellagic acid 4'-O- $\alpha$-rhamnopyranoside (32 mg), arjungenin (12.0 mg), arjunglucoside (3.5 mg), 2 $2,3 \alpha, 24$-trihydroxyolean-11,13(18)-dien-28-oic acid (3.5 mg) and a dark mixture that was subjected to column chromatography on Sephadex LH-20 with $\mathrm{MeOH}$ as an isocratic eluent and yielded 3-O-methyl ellagic acid $(12.5 \mathrm{mg})$. Fraction $\mathrm{T}_{4}(m=56.18 \mathrm{~g})$ obtained with the solvent system of EtOAc-MeOH (85:15 to 65:35) was a complex mixture and thus was not studied. All the isolated compounds were screened as described above for anti-yeast activity, and as described below for enzyme inhibition activities.

\subsection{Purification of Glucose 6-Phosphate Dehydrogenase and Activity Determination}

G6PD was purified from the gill tissue of Lake Van fish according to Kuzu et al. [29], and the enzyme activity was determined spectrophotometrically using a Shimadzu UV-1800 spectrophotometer (Shimadzu, Tokyo, Japan) at $25^{\circ} \mathrm{C}$, according to the method described by Beutler [30] and based on the principle of the reduction of $\mathrm{NADP}^{+}$to NADPH in the presence of glucose 6-phosphate and absorbance recorded at $340 \mathrm{~nm}$.

\subsection{Purification of Carbonic Anhydrase Isoenzymes by Affinity Chromatography and Activity Determination}

The purification of hCA I and II isozymes was performed with a simple one step method by a Sepharose-4B anilinesulphanilamide affinity column chromatograph as previously described [31]. Briefly, CNBr activated Sepharose-4B was washed with $\mathrm{ddH}_{2} \mathrm{O}$ and tyrosine further attached to the activated gel as a spacer arm and finally diazotized sulphanilamide clamped with tyrosine molecule as ligand. The homogenate was applied to the prepared Sepharose-4B-L-Tyrosine Sulphanilamide affinity column equilibrated with $25 \mathrm{mM}$ Tris- $\mathrm{HCl} / 0.1 \mathrm{M} \mathrm{Na}_{2} \mathrm{SO} 4$ (pH 8.7) (Sigma-Aldrich, Taufkirchen, Germany). The affinity gel was washed with $25 \mathrm{mM}$ Tris- $\mathrm{HCl} / 22 \mathrm{mM} \mathrm{Na}_{2} \mathrm{SO} 4$ (pH 8.7).

The esterase activity was assessed following the change in absorbance of 4-nitrophenylacetate (NPA) to 4-nitrophenylate ion at $348 \mathrm{~nm}$ over a period of $3 \mathrm{~min}$ at $25^{\circ} \mathrm{C}$ using a Beckman Coulter UV-VIS spectrophotometer (Beckman Coulter, Atlanta, GA, USA) according to the method described by Verpoorte et al. [32].

\subsection{Purification of Glutathione S-Transferase Enzyme and Activity Determination}

Firstly, heamolysate from human erythrocytes was prepared according to the method of Hunaiti et al. [33]. The prepared heamolysate was directly applied to the glutathione-agarose affinity column and washed with $10 \mathrm{mM} \mathrm{KH}_{2} \mathrm{PO}_{4}$ and $0.1 \mathrm{M} \mathrm{KCl}$ (pH 8.0) (Sigma-Aldrich). The washing procedure was monitored on a spectrophotometer through equal-to-blind absorbance values. After the column was stabilized, the enzyme was purified by gradient elution at $+4{ }^{\circ} \mathrm{C}[24,34]$. Elution solvent was prepared from a solvent gradient containing $50 \mathrm{mM}$ Tris- $\mathrm{HCl}$ and $(1.25-10 \mathrm{mM} \mathrm{GSH}$, $\mathrm{pH}$ 9.5). Thereafter, 1-chloro-2,4-dinitrobenzene (Sigma-Aldrich) was used to determine GST enzyme activity. In fact the complex obtained using dinitrobenzene S-glutathione (DNB-SG) displays maximum 
absorbance at $340 \mathrm{~nm}$. Activity measurements were thus carried out using the absorbance increment at this wavelength. [35].

\subsection{In Vitro Inhibition and Kinetic Studies}

To determine the effects of compounds on enzymes, enzyme activities were measured with saturated substrate concentration and five different inhibitor concentrations. The 50\% inhibitory concentrations $\left(\mathrm{IC}_{50}\right)$ were determined by plotting curves of percent inhibition versus compound concentration. Results are reported as $\mathrm{IC}_{50}$ values. Ki constants were calculated using the Cheng-Prusoff equation [36].

\section{Results and Discussion}

The methanol extract of the stem bark of T. mantaly was screened for anti-yeast activity in vitro against three clinical isolates consisting of C. albicans, C. krusei and C. parapsilosis. The crude extract exhibited good activity with MIC values of $24 \mu \mathrm{g} / \mathrm{mL}$ against $C$. parapsilosis and $39 \mu \mathrm{g} / \mathrm{mL}$ against $C$. albicans and C. krusei (Table 1).

Table 1. Anti-yeast activity of Terminalia mantaly extract and isolates.

\begin{tabular}{|c|c|c|c|c|}
\hline \multirow{2}{*}{\multicolumn{2}{|c|}{ Extract/Fractions }} & C. parapsilosis & C. albicans & C. krusei \\
\hline & & \multicolumn{3}{|c|}{$\mathrm{MIC} *(\mu \mathrm{g} / \mathrm{Ml} \pm \mathrm{SD})$} \\
\hline \multicolumn{2}{|l|}{$\mathrm{MeOH}$ Extract } & $24.00 \pm 0.21$ & $39.00 \pm 0.33$ & $39.00 \pm 0.30$ \\
\hline \multicolumn{2}{|l|}{ Fraction T1 } & $1250.00 \pm 1.23$ & $2500.00 \pm 0.98$ & $2500.00 \pm 1.03$ \\
\hline \multicolumn{2}{|l|}{ Fraction T2 } & $39.00 \pm 0.38$ & $>5000$ & $>5000$ \\
\hline \multicolumn{2}{|l|}{ Fraction T3 } & $0.16 \pm 0.02$ & $0.64 \pm 0.12$ & $0.02 \pm 0.09$ \\
\hline \multicolumn{2}{|l|}{ Fraction T4 } & $>5000$ & $>5000$ & $>5000$ \\
\hline \multicolumn{5}{|c|}{ Fraction of origin } \\
\hline $\begin{array}{l}\text { 3,3'-di-O-methylellagic acid } \\
4^{\prime}-O-\alpha \text {-rhamnopyranoside }\end{array}$ & $\mathrm{T} 3$ & $\begin{array}{l}39.00 \pm 0.88 \\
(80.4 \mu \mathrm{M})\end{array}$ & $\begin{array}{l}9.70 \pm 0.72 \\
(20 \mu \mathrm{M})\end{array}$ & $\begin{array}{c}>5000 \\
(10,300 \mu \mathrm{M})\end{array}$ \\
\hline 3-O-methyl ellagic acid & T3 & $\begin{array}{l}78.00 \pm 0.92 \\
(247.6 \mu \mathrm{M})\end{array}$ & $\begin{array}{l}156.00 \pm 1.00 \\
\quad(495 \mu \mathrm{M})\end{array}$ & $\begin{array}{c}19.50 \pm 0.57 \\
(61.9 \mu \mathrm{M})\end{array}$ \\
\hline Arjungenin & $\mathrm{T} 1, \mathrm{~T} 2, \mathrm{~T} 3$ & $\begin{array}{c}>5000 \\
(9487 \mu \mathrm{M})\end{array}$ & $\begin{array}{c}>5000 \\
(9487 \mu \mathrm{M})\end{array}$ & $\begin{array}{c}>5000 \\
(9487 \mu \mathrm{M})\end{array}$ \\
\hline Arjunglucoside & $\mathrm{T} 2, \mathrm{~T} 3$ & $\begin{array}{c}39.00 \pm 0.13 \\
(56.60 \mu \mathrm{M})\end{array}$ & $\begin{array}{l}9.70 \pm 0.36 \\
(14.07 \mu \mathrm{M})\end{array}$ & $\begin{array}{c}312.00 \pm 1.04 \\
(452 \mu \mathrm{M})\end{array}$ \\
\hline $\begin{array}{c}2 \alpha, 3 \alpha, 24 \text {-trihydroxyolean-11, } \\
13(18) \text {-dien-28-oic acid }\end{array}$ & $\mathrm{T} 1, \mathrm{~T} 3$ & $\begin{array}{c}>5000 \\
(9823 \mu \mathrm{M})\end{array}$ & $\begin{array}{c}>5000 \\
(9823 \mu \mathrm{M})\end{array}$ & $\begin{array}{c}>5000 \\
(9823 \mu \mathrm{M})\end{array}$ \\
\hline Fluconazole $* *$ & & $\begin{array}{l}2.00 \pm 0.01 \\
(6.53 \mu \mathrm{M})\end{array}$ & $\begin{array}{l}8.00 \pm 0.25 \\
(26.14 \mu \mathrm{M})\end{array}$ & $\begin{array}{c}32.00 \pm 0.42 \\
(10.45 \mu \mathrm{M})\end{array}$ \\
\hline
\end{tabular}

* Plant extracts were tested using the CLSI M27-A3 protocol. Activity was expressed as minimal inhibitory concentration; ${ }^{* *}$ Reference used as positive control. MIC, minimum inhibitory concentration.

The flash chromatography of the crude extract generated four fractions exhibiting varying antifungal activities. As shown in Table 1, fraction T3 was the most active, with activity magnification over 1950 times against $C$. krusei $(\mathrm{MIC}=0.02 \mu \mathrm{g} / \mathrm{mL}), 150$ times against $C$. parapsilosis $(\mathrm{MIC}=0.16 \mu \mathrm{g} / \mathrm{mL})$, and over 60 times against C. albicans $(0.64 \mu \mathrm{g} / \mathrm{mL})$, compared to the crude extract $(\mathrm{MIC}=24-39 \mu \mathrm{g} / \mathrm{mL})$. C. krusei was the most susceptible isolate to fraction T3. Compounds 3,3'-di-O-methylellagic acid-4'-O- $\alpha$-rhamnopyranoside, 3-O-methylellagic acid, arjungenin, arjunglucoside, and 2 $\alpha, 3 \alpha, 24$-trihydroxyolean-11,13(18)-dien-28-oic acid that were all found in fraction T3 were also tested for biological activity (Table 1; Figure 1). Overall, they showed drastically reduced potency against the tested yeasts compared to the mother fraction T3, 
indicating that fractionation has negatively affected the biological activity. Compounds stigmasterol and stigmasterol 3-O- $\beta$-D-glucopyranoside were not tested due to reduced solubility in the culture medium. Among the tested compounds, 3,3'-di-O-methylellagic acid- $4^{\prime}-O$ - $\alpha$-rhamnopyranoside and arjunglucoside showed the best potency against C. albicans with an MIC of $9.7 \mu \mathrm{g} / \mathrm{mL}$. They also moderately inhibited $C$. parapsilosis with an MIC of $39 \mu \mathrm{g} / \mathrm{mL}$. In addition, compound 3-O-methylellagic acid inhibited C. krusei with an MIC of $19.5 \mu \mathrm{g} / \mathrm{mL}$.

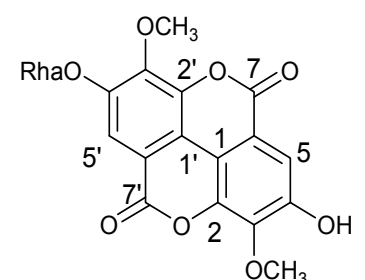

3,3'-di-O-methylellagic acid 4'-O- $\alpha$-rhamnopyranoside<smiles>[R7]c1c(O)cc2c(=O)oc3c(O)c(O)cc4[Z9](=O)oc1c2c43</smiles>

ellagic acid: $\mathrm{R}=\mathrm{H}$ 3-O-methyl ellagic acid: $\mathrm{R}=\mathrm{CH}_{3}$

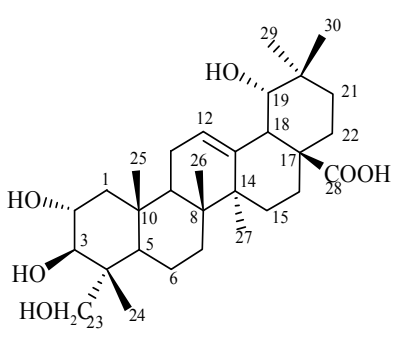

arjungenin

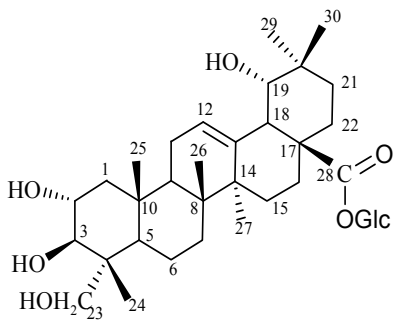

arjunglucoside

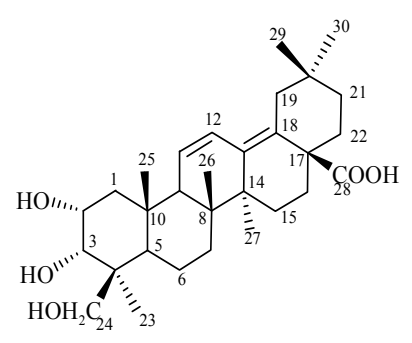

$2 \alpha, 3 \alpha, 24$-trihydroxyolean-11,13(18)-dien-28-oic acid<smiles>CCC(/C=C/[C@@H](C)C1CCC2C3CC=C4C[C@@H](O)CC[C@]4(C)C3CC[C@@]21N)C(C)C</smiles>

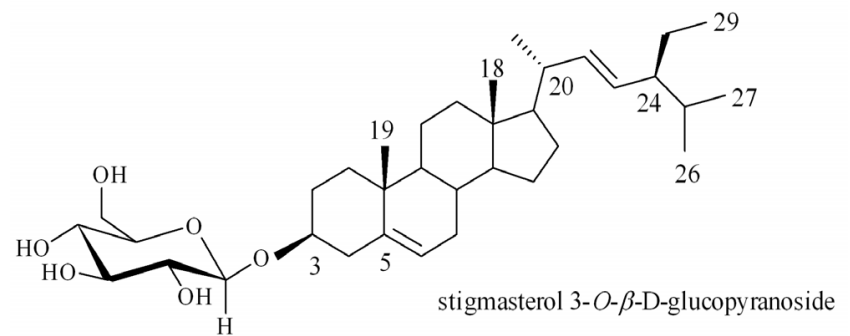

Figure 1. Structures of the isolated compounds from Terminalia mantaly (Combretaceae). The isolated compounds were tested against pathogenic yeast isolates and enzymes of metabolic significance. 3,3'-di-O-methylellagic acid 4'-O- $\alpha$-rhamnopyranoside: $\mathrm{IC}_{50}=39 \mu \mathrm{g} / \mathrm{mL}$ C. parapsilosis; $9.7 \mu \mathrm{g} / \mathrm{mL}$ C. albicans; $>5000 \mu \mathrm{g} / \mathrm{mL}$ C. krusei; CAI: $\mathrm{IC}_{50}=53.31 \mu \mathrm{M}, \mathrm{Ki}=44.11 \mu \mathrm{M}$; CAII: $\mathrm{IC}_{50}=69.11 \mu \mathrm{M}$, $\mathrm{Ki}=55.78 \mu \mathrm{M}$; GST: $\mathrm{IC}_{50}=63.01 \mu \mathrm{M}, \mathrm{Ki}=42.00 \mu \mathrm{M}$. 3-O-methyl ellagic acid: $\mathrm{IC}_{50}=78 \mu \mathrm{g} / \mathrm{mL}$ C. parapsilosis; $156 \mu \mathrm{g} / \mathrm{mL}$ C. albicans; $19.5 \mu \mathrm{g} / \mathrm{mL}$ C. krusei. arjungenin: C. parapsilosis, C. albicans, krusei: $\mathrm{IC}_{50}>5000 \mu \mathrm{g} / \mathrm{mL}$; CAI: $\mathrm{IC}_{50}=86.64 \mu \mathrm{M}, \mathrm{Ki}=71.68 \mu \mathrm{M}$; GST: $\mathrm{IC}_{50}=1.51 \mu \mathrm{M}, \mathrm{Ki}=1.00 \mu \mathrm{M}$; arjunglucoside: $\mathrm{IC}_{50}=39 \mu \mathrm{g} / \mathrm{mL}$ C. parapsilosis; $9.7 \mu \mathrm{g} / \mathrm{mL}$ C. albicans; $312 \mu \mathrm{g} / \mathrm{mL}$ C. krusei; G6PD: $\mathrm{IC}_{50}=1.84 \mu \mathrm{M}, \mathrm{Ki}=0.19 \mu \mathrm{M}$; CAI: $\mathrm{IC}_{50}=3.28 \mu \mathrm{M}, \mathrm{Ki}=2.72 \mu \mathrm{M} ;$ CAII: $\mathrm{IC}_{50}=1.28 \mu \mathrm{M}$, $\mathrm{Ki}=1.03 \mu \mathrm{M}$; GST: $\mathrm{IC}_{50}=1.84 \mu \mathrm{M}, \mathrm{Ki}=1.23 \mu \mathrm{M}$. 2 $\alpha, 3 \alpha, 24$-trihydroxyolean-11,13(18)-dien-28-oic acid: $\mathrm{IC}_{50}>5000 \mu \mathrm{g} / \mathrm{mL}$ C. parapsilosis, C. albicans, C. krusei.

\section{NMR Spectral Data of the Tested Compounds}

The physicochemical profiles of the isolated compounds were acquired following previously described approaches [37-40]. 
3,3'-di-O-methylellagic acid 4'-O- $\alpha$-rhamnopyranoside. Yellowish powder; molecular formula $\mathrm{C}_{21} \mathrm{H}_{18} \mathrm{O}_{12}$; ESI-MS: $[\mathrm{M}+\mathrm{Na}]^{+} m / z$ 485,049 ${ }^{1} \mathrm{H}-\mathrm{NMR}\left(300 \mathrm{MHz}, \mathrm{DMSO}-d_{6}\right): \delta_{\mathrm{H}} 1.13\left(3 \mathrm{H}, \mathrm{d}, \mathrm{CH}_{3}, \mathrm{H}-6^{\prime \prime}\right), 3.54(1 \mathrm{H}$, q, $J=8.0$ and $\left.12.0 \mathrm{~Hz}, \mathrm{H}-5^{\prime \prime}\right), 4.01\left(1 \mathrm{H}, \mathrm{t}, \mathrm{H}-4^{\prime \prime}\right), 4.04(3 \mathrm{H}, \mathrm{s}, \mathrm{OMe}-3), 4.72\left(1 \mathrm{H}, \mathrm{brd}, J=8.0 \mathrm{~Hz}, \mathrm{H}-3^{\prime \prime}\right)$, $4.94\left(1 \mathrm{H}, \mathrm{brd}, J=4.0 \mathrm{~Hz}, \mathrm{H}-2^{\prime \prime}\right), 5.47\left(1 \mathrm{H}, \mathrm{brs}, \mathrm{H}-\mathrm{-}^{\prime \prime}\right), 7.52(1 \mathrm{H}, \mathrm{s}, \mathrm{H}-5), 7.73\left(1 \mathrm{H}, \mathrm{s}, \mathrm{H}-5^{\prime}\right) ;{ }^{13} \mathrm{C}-\mathrm{NMR}$ (125 MHz, DMSO- $\left.d_{6}\right)$, aglycone moiety: $\delta_{\mathrm{C}} 113.4$ (C-1), 140.5 (C-2), 141.8 (C-3), 153.1 (C-4), 111.9 (C-5), $113.4(\mathrm{C}-6), 159.1(\mathrm{C}-7), 114.7\left(\mathrm{C}-1^{\prime}\right), 136.6\left(\mathrm{C}-2^{\prime}\right), 142.2\left(\mathrm{C}-3^{\prime}\right), 146.9\left(\mathrm{C}-4^{\prime}\right), 112.0\left(\mathrm{C}-5^{\prime}\right), 107.4\left(\mathrm{C}-6^{\prime}\right)$, $159.1\left(\mathrm{C}-7^{\prime}\right)$; rhamnose moiety: $100.5\left(\mathrm{C}-1^{\prime \prime}\right), 70.4\left(\mathrm{C}-2^{\prime \prime}\right), 70.5\left(\mathrm{C}-3^{\prime \prime}\right), 72.2\left(\mathrm{C}-4^{\prime \prime}\right), 70.3\left(\mathrm{C}-5^{\prime \prime}\right), 18.3\left(\mathrm{C}-6^{\prime \prime}\right)$ and $61.4(\mathrm{C}-3, \mathrm{OMe})$.

3-O-methyl ellagic acid. Yellowish powder; molecular formula $\mathrm{C}_{15} \mathrm{H}_{8} \mathrm{O}_{8}$; ESI-MS: $[\mathrm{M}-\mathrm{H}]^{-} \mathrm{m} / z$ 315, ${ }^{1} \mathrm{H}-\mathrm{NMR}\left(\mathrm{DMSO}-d_{6}\right): \delta 7.50(1 \mathrm{H}, \mathrm{s}, \mathrm{H}-5), 7.44\left(1 \mathrm{H}, \mathrm{s}, \mathrm{H}-5^{\prime}\right), 4.02(3 \mathrm{H}, \mathrm{s}, 3-\mathrm{OMe}) .{ }^{13} \mathrm{C}-\mathrm{NMR}$ (DMSO- $\left.d_{6}\right)$ : $\delta 158.9(\mathrm{C}-7), 158.6\left(\mathrm{C}-7^{\prime}\right), 152.2(\mathrm{C}-4), 148.2\left(\mathrm{C}-4^{\prime}\right), 141.7(\mathrm{C}-2), 140.0(\mathrm{C}-3), 139.8\left(\mathrm{C}-3^{\prime}\right), 136.1\left(\mathrm{C}-2^{\prime}\right)$, 112.4(C-1'), 112.1 (C-6), 111.7 (C-1), 111.3 (C-5), $110.1\left(\mathrm{C}-5^{\prime}\right), 107.2\left(\mathrm{C}-6^{\prime}\right), 60.8$ (3-OMe).

Arjungenin or 2,3,19,23-tetrahydroxyolean-12-en-28-oic acid. White powder; molecular formula $\mathrm{C}_{30} \mathrm{H}_{48} \mathrm{O}_{6}$; ESI-MS: $[\mathrm{M}+\mathrm{Na}]^{+} m / z 527,322 .{ }^{1} \mathrm{H}-\mathrm{NMR}\left(300 \mathrm{MHz}, \mathrm{DMSO}-d_{6}\right): \delta_{\mathrm{H}} 1.23,1.09,0.90,0.88,0.84$ and 0.65 (each $3 \mathrm{H}, \mathrm{s}) ; 2.92(1 \mathrm{H}, \mathrm{brs}, \mathrm{H}-18) ; 2.86(1 \mathrm{H}, \mathrm{d}, J=8 \mathrm{~Hz}, \mathrm{H}-3)$ and $3.57(1 \mathrm{H}, \mathrm{m}, \mathrm{H}-2) ; 5.23(1 \mathrm{H}$, brs, H-12); ${ }^{13} \mathrm{C}-\mathrm{NMR}\left(125 \mathrm{MHz}\right.$; DMSO- $\left.d_{6}\right): \delta_{\mathrm{C}} 16.8,17.1,23.9,24.9,28.9$ and $24.5 ; 64.3(\mathrm{C}-23), 80.5(\mathrm{C}-3)$, 179.6 (C-28), 122.6 (C-12); 143.9 (C-13).

Arjunglucoside or 2,3,19,23-tetrahydroxyolean-12-en-28-ö̈ acid glucopyranoside. White powder; molecular formula $\mathrm{C}_{36} \mathrm{H}_{58} \mathrm{O}_{11}$; ESI-MS: [M + Na] ${ }^{+} m / z 689,396 ;{ }^{1} \mathrm{H}-\mathrm{NMR}$ (300 MHz, DMSO- $d_{6}$ ): $\delta 1.23,1.08,0.89$, $0.86,0.84$ and 0.63 (each $3 \mathrm{H}, \mathrm{s}$ ); between 2.90 and 3.80: glucose moiety with anomeric proton at 5,20 $\left(1 \mathrm{H}, \mathrm{d}, J=6.9 \mathrm{~Hz}, \mathrm{H}-1^{\prime}\right) ;{ }^{13} \mathrm{C}-\mathrm{NMR}\left(125 \mathrm{MHz}\right.$; DMSO- $\left.d_{6}\right): \delta 16.9,24.5,24.9$ and 28.5; glucose moiety: 61.0, 69.9, 72.8, 77.1, 78.2, 94.5; 64.3 (C-23), 67.4 (C-2), 80.4 (C-3), 176.3 (C-28), 122.6 (C-12), 143.7 (C-13).

$2 \alpha, 3 \alpha, 24-t r i h y d r o x y o l e a n-11,13(18)$-dien-28-oic acid. Yellowish powder; molecular formula $\mathrm{C}_{30} \mathrm{H}_{46} \mathrm{O}_{5}$; ESI-MS: $[\mathrm{M}+\mathrm{Na}]^{+} m / z 509,375$ (calc. 509,324$)$ for $\left.\mathrm{C}_{30} \mathrm{H}_{46} \mathrm{NaO}_{5}\right) ;{ }^{1} \mathrm{H}-\mathrm{NMR}\left(400 \mathrm{MHz}\right.$; pyridin- $\left.d_{5}\right): \delta$ $1.58 ; 1.06 ; 1.05 ; 1.03 ; 0.90$ and $0.87($ each $3 \mathrm{H}, \mathrm{s}) ; 6.62(1 \mathrm{H}, \mathrm{d}, J=8.0 \mathrm{~Hz}, \mathrm{H}-11)$ and $5.81(1 \mathrm{H}, \mathrm{d}, J=8.0$ $\mathrm{Hz}, \mathrm{H}-12) ; 4.38(1 \mathrm{H}, \mathrm{ddd}, J=2.2 ; 7.6$ and $8.9 \mathrm{~Hz}, \mathrm{H}-2) ; 3.59(1 \mathrm{H}, \mathrm{d}, J=7.5 \mathrm{~Hz}, \mathrm{H}-3) ; 4.43(1 \mathrm{H}, \mathrm{d}, J=8.7$ $\mathrm{Hz}, \mathrm{H}-24)$ and $3.75(1 \mathrm{H}, \mathrm{d}, J=8,76 \mathrm{~Hz}, \mathrm{H}-24) ; 2.69(1 \mathrm{H}, \mathrm{d}, J=12.4 \mathrm{~Hz}, \mathrm{H}-19)$ and $2.15(1 \mathrm{H}, \mathrm{d}, J=12.4$ Hz, H-19); ${ }^{13} \mathrm{C}-\mathrm{NMR}\left(125 \mathrm{MHz}\right.$; pyridin- $\left.d_{5}\right): \delta_{\mathrm{C}} 16.6,19.5,19.8,23.7,24.0$ and $32.1 ; 65.1$ (C-24), 68.4 (C-2), 85.5 (C-3), 178,6 (C-28);136,1 (C-13); 133,3 (C-18); 126,4 (C-12) et 125,9 (C-11).

Apart from the activity profile described above, MIC values for the other tested fractions and compounds were above $39 \mu \mathrm{g} / \mathrm{ml}$. The activity level of compounds 3,3'-di-O-methylellagic acid $4^{\prime}-O-\alpha$-rhamnopyranoside and arjunglucoside was comparable to that of the reference drug fuconazole against $\mathrm{C}$. albicans, and compound 3-O-methylellagic acid showed to be over 1.5 times more active than the same reference drug against $C$. krusei. Based on the basic skeleton of the tested compounds, it is important to notice that one of the most active derivatives, arjunglucoside and the less active compounds, arjungenin and $2 \alpha, 3 \alpha, 24$-trihydroxyolean-11,13(18)-dien-28-oic acid are all triterpenoids. Preliminary structure-activity relationship (SAR) study clearly indicated that the glycosylation of the acidic function of arjungenin at C-28 is important for activity improvement. The other active compounds 3,3'-di-O-methylellagic acid $4^{\prime}-O-\alpha$-rhamnopyranoside and 3-O-methylellagic acid are ellagic acid derivatives. Previous studies have reported the antifungal activity of ellagic acid against fungal strains Trichophyton rubrum, T. verrucosum, T. mentagrophytes, T. violaceum, T. schoenleinii, Microsporum canis, C. glabrata, C. albicans and C. tropicalis [41]. Also, the observed antifungal potency of compounds 3,3'-di-O-methylellagic acid $4^{\prime}-O-\alpha$-rhamnopyranoside and 3-O-methylellagic acid, respectively glycosylated and methylated derivatives of ellagic acid, highlights the potency of this class of secondary metabolites [41].

Overall, it was observed that fraction T3 exerted the more potent effect against the tested yeasts, far better than the derived compounds. This is an indication that fractionation has declined the anti-yeast activity, emphasizing the relevance of potential synergistic interactions among the components of 
fraction T3. Moreover, these results indicate future directions in the progression of this fraction to develop a phytodrug against yeasts infections.

Selected isolated compounds were further tested against G6PD, carbonic anhydrase I, II and GST enzymes. The results achieved are shown in Table 2.

The G6PD enzyme was strongly inhibited by the triterpenoid arjunglucoside with $\mathrm{IC}_{50}$ value of $1.84 \mu \mathrm{M}$ and $\mathrm{Ki}$ (the inhibitor constant indicating how potent an inhibitor is; or the concentration required to produce half maximum inhibition) value of $0.19 \mu \mathrm{M}$. It has been shown that this key metabolic enzyme which catalyzes the first step of the pentose phosphate pathway is expressed abundantly and is very active in human tumors [21]. In contrast, G6PD-deficient tumor cell lines showed relatively slow growth and enhanced apoptosis [42]. Previous studies also reported G6PD inhibitory properties for few compounds such as steroids and derivatives [43,44], chalcones [29], catechin gallates [45], and some phenolic molecules [46]. In this study, the substituted ellagic acid derived compound 3,3'-di-O-methylellagic acid $4^{\prime}-O-\alpha$-rhamnopyranoside did not show any effect on the G6PD enzyme activity, although Adem et al. [46] had previously reported that ellagic acid inhibited the enzyme with an $\mathrm{IC}_{50}$ value of $0.072 \mathrm{mM}$. The methoxy group in this derivative may hinder the enzyme-inhibitor interaction. Based on the skeletal features of the tested triterpenoids-arjungenin, arjunglucoside, and 2 $\alpha, 3 \alpha, 24$-trihydroxyolean-11,13(18)-dien-28-oic acid-the presence of the hydroxyl group at C-19 and the glycosylation of the C-28 carboxylic group may be both factors of activity improvement. The G6PD inhibitory potential of a terpenoid is reported here for the first time.

Compound arjunglucoside exhibited very good potency against both CAI and CAII enzymes with respective activity parameters of $\mathrm{IC}_{50}=3.28 \mu \mathrm{M}$ and $\mathrm{Ki}=2.72 \mu \mathrm{M}$; and $\mathrm{IC}_{50}=1.28 \mu \mathrm{M}$ and $\mathrm{Ki}=1.03 \mu \mathrm{M}$ respectively. The other tested compounds including 3,3'-di-O-methylellagic acid $4^{\prime}-O-\alpha$-rhamnopyranoside and arjungenin were found to be moderately active against CAI and CAII (3,3'-di-O-methylellagic acid $4^{\prime}$-O- $\alpha$-rhamnopyranoside) with $\mathrm{IC}_{50}$ and $\mathrm{Ki}$ values globally above $44 \mu \mathrm{M}$. Previous studies by Sarkkaya et al. [47] have indicated that ellagic acid inhibited CAI and CAII with $K_{i}$ values of 0.207 and $0.146 \mathrm{mM}$ respectively. In the present study, compound 3,3'-di-O-methylellagic acid $4^{\prime}$-O- $\alpha$-rhamnopyranoside, a substituted derivative of ellagic acid has exhibited moderate, however highly improved potency toward CAI $\left(K_{i}=44.11 \mu \mathrm{M}\right)$ and CAII $(55.78 \mu \mathrm{M})$ enzymes. On the other hand, this substitution has also considerably decreased the activity as observed against the G6PD enzyme. In addition to the established role of CA inhibitors (CAIs) as diuretics and antiglaucoma drugs, it has recently emerged that they could have potential as novel anti-obesity, anticancer and anti-infective drugs [23]. The high inhibitory potency of the triterpenoid arjunglucoside against CAs indicates that it is a promising compound that might be a candidate for the formulation of drugs against CAIs-related diseases.

The screening of 3,3'-di-O-methylellagic acid $4^{\prime}-O-\alpha$-rhamnopyranoside, arjungenin, and arjunglucoside against GST enzyme showed inhibitory effects. However, the triterpenoids arjungenin, and arjunglucoside exhibited highly potent inhibitory effects $\left(\mathrm{IC}_{50}\right.$ of 1.57 and $1.84 \mu \mathrm{M}$ respectively; and $K_{i}$ of 1.00 and $1.23 \mu \mathrm{M}$ respectively). Compound 3,3'-di-O-methylellagic acid $4^{\prime}-\mathrm{O}-\alpha$-rhamnopyranoside only exerted a moderate inhibitory effect on the enzyme $\left(\mathrm{IC}_{50}=63.01 \mu \mathrm{M}\right.$; $\left.K_{i}=42.00 \mu \mathrm{M}\right)$. These results are of higher significance as GST inhibitors are anti-cancer agents $[25,26]$. Ellagic acid was recently shown to inhibit GSTs A1-1, A2-2, M1-1, M2-2 and P1-1 with IC 50 values ranging from 0.04 to $5 \mu \mathrm{M}$ [48]. Preliminary SAR studies indicate that the substitution of ellagic acid at C-3 and C-4' gave the derivative 3, $3^{\prime}$-di-O-methylellagic acid $4^{\prime}-O-\alpha$-rhamnopyranoside which showed an $\mathrm{IC}_{50}$ value of $63.01 \mu \mathrm{M}$, thus therefore considerably decreased the activity. The inhibitory effect of this class of secondary metabolite derivatives is reported here for the first time. 
Table 2. Inhibitory parameters of isolated compounds against G6PD, CAI, CAII, and GST.

\begin{tabular}{|c|c|c|c|c|c|c|c|c|c|c|c|c|c|c|c|}
\hline \multirow{2}{*}{$\begin{array}{c}\text { Activity } \\
\text { Parameter }\end{array}$} & \multicolumn{4}{|c|}{ G6PD } & \multicolumn{3}{|c|}{ CAI } & \multicolumn{4}{|c|}{ CAII } & \multicolumn{4}{|c|}{ GST } \\
\hline & 1 & 2 & 3 & 4 & 1 & 3 & 4 & 1 & 2 & 3 & 4 & 1 & 2 & 3 & 4 \\
\hline $\mathrm{IC}_{50}{ }^{\mathrm{a}}(\mu \mathrm{M})$ & n.a & n.a & n.a & $1.84 \pm 0.31$ & $53.31 \pm 1.09$ & $86.64 \pm 0.93$ & $3.28 \pm 0.13$ & $69.31 \pm 1.13$ & n.a & n.a & $1.03 \pm 0.01$ & $63.01 \pm 1.15$ & n.a & $1.51 \pm 0.78$ & \\
\hline $\mathrm{Ki}^{\mathrm{b}}(\mu \mathrm{M})$ & n.a & n.a & n.a & $0.19 \pm 0.03$ & $44.11 \pm 1.12$ & $71.68 \pm 0.96$ & $2.72 \pm 0.64$ & $55.78 \pm 0.97$ & n.a & n.a & $1.84 \pm 0.11$ & $42.00 \pm 1.39$ & n.a & $1.00 \pm 0.03$ & $0.19 \pm 0.77$ \\
\hline
\end{tabular}

Enzymes were expressed and purified, and subsequently assessed for in vitro susceptibility to inhibitors. ${ }^{\text {a }}$ Serially diluted triplicate concentrations of compounds were tested and activity expressed as $50 \%$ inhibitory concentration; ${ }^{\mathrm{b}}$ Inhibitory constant which is reflective of the binding affinity; the smaller the Ki, the greater the binding affinity and the smaller amount of medication needed in order to inhibit the activity of that enzyme. n.a = non active. 1: 3,3'-di-O-methylellagic acid $4^{\prime}$-O- $\alpha$-rhamnopyranoside; 2 : 3-O-methylellagic acid 3: arjungenin; 4: arjunglucoside. G6PD, glucose-6-phosphate dehydrogenase; CAI, human erythrocyte carbonic anhydrase I; CAII, human erythrocyte carbonic anhydrase II; GST, glutathione $S$-transferase. 


\section{Concluding Remarks}

The results obtained from the investigation of the methanolic extract of T. mantaly stem bark have identified a highly potent anti-yeast fraction T3 that showed to be more promising than subsequently isolated compounds. Overall, the five compounds, 3,3'-di-O-methylellagic acid $4^{\prime}$-O- $\alpha$-rhamnopyranoside, arjungenin, arjunglucoside, $2 \alpha, 3 \alpha, 24$-trihydroxyolean-11,13(18)-dien-28-oic acid, and 3-O-methyl ellagic acid were found to be 243 to 31,250 times, 15 to 7,812 times, and 975 to 250,000 times less active than the mother fraction (T3) against C. parapsilosis, C. albicans, and C. krusei respectively. This promising fraction deserves to be further investigated with the ultimate aim of formulating a plant-based drug against yeast infections. Compounds 3,3'-di-O-methylellagic acid $4^{\prime}-O-\alpha$-rhamnopyranoside and arjunglucoside showed anti-yeast activity close to that of the reference drug fuconazole against $C$. albicans. Moreover, compound 3-O-methyl ellagic acid was over 1.5 times more active than fuconazole against $C$. krusei. In addition, two of the islolated compounds, arjungenin and arjunglucoside were found to be very active against enzymes of metabolic significance, namely G6PD (arjunglucoside) and GST (arjungenin and arjunglucoside). Finally, given the anti-yeast potency of these compounds, and also the implication of the tested enzymes in some metabolic dysfunctions of public health significance (cancer, obesity, epilepsy), we envisage further SAR studies to identify potent hit derivatives that should subsequently enter the drug development pipeline.

Acknowledgments: The authors acknowledge the Alexander von Humboldt Foundation for providing a Fellowship to Bruno Ndjakou Lenta at Bielefeld University. Part of this study was supported by equipment from the Seeding Labs' Instrumental Access Grant (SL2012-2) to Fabrice Fekam Boyom.

Author Contributions: N.S., E.T., B.N.L., F.F.B. and S.A.N. designed and supervised the study; M.A.T.T., T.N.K., S.G., R.M.T.K., A.A., M.K., V.C. and R.D. performed the chemical and biological parts of the study and drafted the manuscript; B.N.L. and F.F.B. critically revised the manuscript. All authors agreed on the final version of the manuscript for submission to Medicines.

Conflicts of Interest: The authors declare no conflict of interest.

\section{References}

1. Arif, T.; Mandal, T.K.; Dabur, R. Natural products: Anti-fungal agents derived from Plants. In Opportunity, Challenge and Scope of Natural Products in Medicinal Chemistry; Pandalai, S.G., Ed.; Research Signpost: Trivandrum, India, 2011; pp. 283-311.

2. Nelesh, G. HIV-associated opportunistic fungal infections: A guide to using the clinical microbiology laboratory. South. Arf. J. HIV Med. 2007, 1, 18-23.

3. Álvaro-Meca, A.; Jensen, J.; Micheloud, D.; Asunción, D.; Gurbindo, D.; Resino, S. Rate of candidiasis among HIV infected children in Spain in the era of highly active antiretroviral therapy (1997-2008). BMC Inf. Dis. 2013, 13, 1-15. [CrossRef] [PubMed]

4. Brissaud, O.; Guichoux, J.; Harambat, J.; Tandonnet, O.; Zaoutis, T. Invasive fungal disease in PICU: Epidemiology and risk factors. Ann. Intensiv. Care 2012, 2. [CrossRef] [PubMed]

5. Ngouana, K.T.; Krasteva, D.; Drakulovski, P.; Toghueo, K.R.; Kouanfack, C.; Ambe, A.; Reynes, J.; Delaporte, E.; Boyom, F.F.; Mallié, M.; et al. Investigation of minor species Candida africana, Candida stellatoidea, and Candida dubliniensis in the Candida albicans complex among Yaoundé (Cameroon) HIV-infected patients. Mycoses 2015, 58, 33-39. [CrossRef] [PubMed]

6. Ngouana, K.T.; Dongtsa, J.; Kouanfack, C.; Tonfack, C.; Foména, S.; Krasteva, D.; Drakulovski, P.; Aghokeng, A.; Mallié, M.; Delaporte, E.; et al. Cryptoccocal meningitis in Yaoundé (Cameroon) HIV infected patients: Diagnosis, frequency and susceptibility of Cryptococcus neoformans isolates to fluconazole. J. Mycol. Med. 2015, 25, 11-16. [CrossRef] [PubMed]

7. Newman, D.J.; Cragg, G.M. Natural products as sources of new drugs over the last 25 years. J. Nat. Prod. 2007, 70, 461-477. [CrossRef] [PubMed]

8. Coulibaly, K. Evaluation of the Antifungal Activity of Extracts of Bark of Commercial Species, Category P1 the Forest of Mopri, Tiassalé (Southern Ivory Coast). Master's Thesis, University of Cocody-Abidjan, Abidjan, Côte D'Ivoire, 2006; pp. 23-25. 
9. Ngouana, T.K.; Mbouna, C.D.J.; Kuipou, R.M.T.; Tchuenmogne, M.A.T.; Zeuko'o, E.M.; Ngouana, V.; Mallié, M.; Bertout, S.; Boyom, F.F. Potent and Synergistic Extract Combinations from Terminalia Catappa, Terminalia Mantaly and Monodora tenuifolia against Pathogenic Yeasts. Medicines 2015, 2, 220-235. [CrossRef]

10. Cock, I. The medicinal properties and phytochemistry of plants of the genus Terminalia (Combretaceae). Inflammopharmacology 2015, 23, 203-229. [CrossRef] [PubMed]

11. Valsaraj, R.; Pushpangadan, P.; Smitt, U.W.; Adsersen, A.; Christensen, S.B.; Sittie, A.; Nyman, U.; Nielsen, C.; Olsen, C.E. New anti-HIV-1, antimalarial and antifungal compounds from Terminalia bellerica. J. Nat. Prod. 1997, 60, 739-742. [CrossRef] [PubMed]

12. Srivastava, S.K.; Srivastava, S.D.; Chouksey, B.K. New constituents of Terminalia alata. Fitoterapia 1999, 70, 390-394. [CrossRef]

13. Conrad, J.; Vogler, B.; Klaiber, I.; Roos, G.; Walter, U.; Kraus, W. Two triterpene esters from Terminalia macroptera bark. Phytochemistry 1998, 48, 647-650. [CrossRef]

14. Conrad, J.; Vogler, B.; Reeb, S.; Klaiber, I.; Papajewski, S.; Roos, G.; Vasquez, E.; Setzer, M.C.; Kraus, W. Isoterchebulin and 4,6-O-isoterchebuloyl-D-glucose, Novel Hydrolyzable Tannins from Terminalia macroptera. J. Nat. Prod. 2001, 64, 294-299. [CrossRef] [PubMed]

15. Kandil, F.E.; Nassar, M.I. A tannin anti-cancer promotor from Terminalia arjuna. Phytochemistry 1998, 47, 1567-1568. [CrossRef]

16. Mahato, S.B.; Nandy, A.K.; Kundu, A.H. Pentacyclic triterpenoids sapogenols and their glycosides from Terminalia bellerica. Tetrahedron 1992, 48, 2483-2484. [CrossRef]

17. Singh, D.V.; Verma, R.K.; Singh, C.S.; Gupta, M.M. RP-LC determination of oleane derivatives in Terminalia arjuna. J. Pharm. Biomed. Anal. 2002, 28, 447-452. [CrossRef]

18. Garcez, R.F.; Garcez, S.W.; Santana, A.L.B.D.; Alves, M.M.; Matos, M.F.C.C.; Scaliante, A.M. Bioactive flavonoids and triterpenes from Terminalia fagifolia (Combretaceae). J. Braz. Chem. Soc. 2006, 17, 1223-1228. [CrossRef]

19. Yeh, G.C.; Daschner, P.J.; Lopaczynska, J.; MacDonald, C.J.; Ciolino, H.P. Modulation of glucose-6-phosphate dehydrogenase activity and expression is associated with aryl hydrocarbon resistance in vitro. J. Biol. Chem. 2001, 276, 34708-34713. [CrossRef] [PubMed]

20. Matsubara, S.; Kato, T.; Oshikawa, K.; Yamada, T.; Takayama, T.; Koike, T.; Sato, I. Glucose-6-phosphate dehydrogenase in rat lung alveolar epithelial cells. An ultrastructural enzyme-cytochemical study. Eur. J. Histochem. 2002, 46, 243-248. [CrossRef] [PubMed]

21. Preuss, J.; Richardson, A.D.; Pinkerton, A.; Hedrick, M.; Sergienko, E.; Rahlfs, S.; Bode, L. Identification and characterization of novel human glucose-6-phosphate dehydrogenase inhibitors. J. Biomol. Screen. 2013, 18, 286-297. [CrossRef] [PubMed]

22. Tripp, B.C.; Smith, K.; Ferry, J.G. Mini review: Carbonic anhydrase: New insights for an ancient enzyme. J. Biol. Chem. 2001, 276, 48615-48618. [CrossRef] [PubMed]

23. Supuran, C.T. Carbonic anhydrases: Novel therapeutic applications for inhibitors and activators. Nat. Rev. Drug Discov. 2008, 7, 168-181. [CrossRef] [PubMed]

24. Comakli, V.; Ciftci, M.; Kufrevioglu, O.I. Effects of some metal ions on rainbow trout erythrocytes glutathione S-transferase enzyme: An in vitro study. J. Enzyme Inhib. Med. Chem. 2013, 28, 1261-1266. [CrossRef] [PubMed]

25. Mannervik, B.; Danielson, U.H. Glutathione transferases-Structure and catalitic activity. CRC Crit. Rev. Biochem. 1988, 23, 283-337. [CrossRef] [PubMed]

26. Matshushita, N.; Aritake, K.; Takada, A.; Hizue, M.; Hayashi, K.; Mitsui, K. Pharmacological studies on the novel antiallergenic drug HQL-79: 2. Elucidation of mechanisms for antiallergic and antiasthmatic effects. Jpn. J. Pharmacol. 1998, 78, 11-22. [CrossRef]

27. Hayes, J.D.; McLellan, L.I.; Stockman, P.K.; Chalmers, J.; Beckett, G.J. Glutathione S-transferases in man: The relationship between rat and human enzymes. Biochem. Soc. Trans. 1987, 15, 721-725. [CrossRef] [PubMed]

28. Mga'ning, B.M.; Lenta, B.N.; Noungoue, D.T.; Antheaume, C.; Fongang, Y.F.; Ngouela, S.A.; Boyom, F.F.; Rosenthal, P.J.; Tsamo, E.; Sewald, N.; et al. Antiplasmodial sesquiterpenes from the seeds of Salacia longipes var. camerunensis. Phytochemistry 2013, 96, 347-352. [CrossRef] [PubMed] 
29. Kuzu, M.; Aslan, A.; Ahmed, I.; Comakli, V.; Demirdag, R.; Uzun, N. Purification of glucose-6-phosphate dehydrogenase and glutathione reductase enzymes from the gill tissue of Lake Van fish and analyzing the effects of some chalcone derivatives on enzyme activities. Fish Physiol. Biochem. 2016, 42, 483-491. [CrossRef] [PubMed]

30. Beutler, E. Red Cell Metabolism: A Manual of Biochemical Methods, 3rd ed.; Grune and Stratton Inc.: Orlando, FL, USA, 1984; pp. 68-70.

31. Ekinci, D.; Cavdar, H.; Talaz, O.; Sentürk, M.; Supuran, C.T. NO-releasing esters show carbonic anhydrase inhibitory action against human isoforms I and II. Bioorg. Med. Chem. 2010, 18, 3559-3563. [CrossRef] [PubMed]

32. Verpoorte, J.A.; Mehta, S.; Edsall, J.T. Esterase activities of human carbonic anhydrases B and C. J. Biol. Chem. 1976, 242, 4221-4229.

33. Hunaiti, A.A.; Soud, M. Effect of lead concentration on the level of glutathione, glutathione $S$-transferase, reductase and peroxidase in human blood. Sci. Total Environ. 2000, 248, 45-50. [CrossRef]

34. Güvercin, S.; Erat, M.; Sakiroglu, H. Determination of some kinetic and characteristic properties of glutathione S-transferase from bovine erythrocytes. Protein Pept. Lett. 2008, 15, 6-12. [PubMed]

35. Habig, W.H.; Pabst, M.J.; Jakoby, W.B. Glutathione $S$-transferases. The first enzymatic step in mercapturic acid formation. J. Biol. Chem. 1974, 249, 7130-7139. [PubMed]

36. Cheng, Y.; Prusoff, W.H. Relationship between the inhibition constant (K1) and the concentration of inhibitor which causes 50 per cent inhibition (I50) of an enzymatic reaction. Biochem. Pharmacol. 1973, 22, 3099-3108. [PubMed]

37. Liu, M.; Katerere, R.D.; Gray, I.A.; Seidel, V. Phytochemistry and antifungal studies on Terminalia mollis and Terminalia brachystemina. Fitoterapia 2009, 80, 369-373. [CrossRef] [PubMed]

38. Nandy, A.K.; Podder, G.; Sahu, N.P.; Mahato, S.B. Triterpenoids and their glycosides from Terminalia bellerica. Phytochemistry 1989, 28, 2769-2772. [CrossRef]

39. Jossang, A.; Seuleiman, M.; Maidou, E.; Bodo, B. Pentacyclic triterpenes from Combretum nigricans. Phytochemistry 1996, 41, 591-594. [CrossRef]

40. Kojima, H.; Tominaga, H.; Sato, S.; Ogura, H. Pentacyclic triterpenoids from Prunella vulgaris. Phytochemistry 1987, 26, 1107-1111. [CrossRef]

41. Li, Z.; Guo, X.; Dawuti, G.; Aibai, S. Antifungal activity of ellagic acid in vitro and in vivo. Phytother. Res. 2015, 29, 1019-1025. [CrossRef] [PubMed]

42. Kuo, W.Y.; Lin, J.Y.; Tang, K. Human glucose-6-phophate dehydrogenase (G6PD). Gene transforms NIH 3T3 cells and induces tumors in nude mice. Int. J. Cancer 2000, 85, 857-864. [CrossRef]

43. Gupta, S.; Cordeiro, T.A.; Michels, P.A.M. Glucose-6-phosphate dehydrogenase is the target for the trypanocidal action of human steroids. Mol. Biochem. Parasitol. 2011, 176, 112-115. [CrossRef] [PubMed]

44. Hamilton, M.N.; Dawson, M.; Fairweather, E.E.; Hamilton, S.N.; Hitchin, R.J.; James, I.D.; Jones, D.S.; Jordan, M.A.; Lyones, J.A.; Small, F.H. Novel steroid inhibitors of glucose 6-phosphate dehydrogenase. J. Med. Chem. 2012, 55, 4431-4445. [CrossRef] [PubMed]

45. Shin, E.S.; Park, J.; Shin, J.M.; Cho, D.; Cho, S.Y.; Shin, D.W.; Ham, M.; Kim, J.B.; Lee, T.R. Cathechin gallates are $\mathrm{NADP}^{+}$-competitive inhibitors of glucose-6-phosphate dehydrogenase and other enzymes that employ $\mathrm{NADP}^{+}$as a coenzyme. Bioorg. Med. Chem. 2008, 16, 3580-3586. [CrossRef] [PubMed]

46. Adem, S.; Comakli, V.; Kuzu, M.; Demirdag, R. Investigation of the effects of some phenolic compounds on the Activities of glucose-6-phosphate dehydrogenase and 6-Phosphogluconate dehydrogenase from human erythrocytes. J. Biochem. Mol. Toxicol. 2014, 28, 510-514. [CrossRef] [PubMed]

47. Beyza, Ö.S.; Gülçin, İ.; Supuran, C.T. Carbonic anhydrase inhibitors: Inhibition of human erythrocyte isozymes I and II with a series of phenolic acids. Chem. Biol. Drug Des. 2010, 75, 515-520. [CrossRef] [PubMed]

48. Hayeshi, R.; Mutingwende, I.; Mavengere, W.; Masiyanise, V.; Mukanganyama, S. The inhibition of human glutathione $S$-transferases activity by plant polyphenolic compounds ellagic acid and curcumin. Food Chem. Toxicol. 2007, 45, 286-295. [CrossRef] [PubMed]

(C) 2017 by the authors; licensee MDPI, Basel, Switzerland. This article is an open access article distributed under the terms and conditions of the Creative Commons Attribution (CC BY) license (http:/ / creativecommons.org/licenses/by/4.0/). 